\title{
THE BLOW-UP SURFACE FOR NONLINEAR WAVE EQUATIONS WITH SMALL SPATIAL VELOCITY
}

\author{
AVNER FRIEDMAN AND LUC OSWALD
}

\begin{abstract}
Consider the Cauchy problem for $u_{t t}-\varepsilon^{2} \Delta u=f(u)$ in space dimension $\leq 3$ where $f(u)$ is superlinear and nonnegative. The solution blows up on a surface $t=\phi_{\varepsilon}(x)$. Denote by $t=\phi(x)$ the blow-up surface corresponding to $v^{\prime \prime}=f(v)$. It is proved that $\left|\phi_{\varepsilon}(x)-\phi(x)\right| \leq C \varepsilon^{2},\left|\nabla\left(\phi_{\varepsilon}(x)-\phi(x)\right)\right| \leq C \varepsilon^{2}$ in a neighborhood of any point $x_{0}$ where $\phi\left(x_{0}\right)<\infty$.
\end{abstract}

1. The main results. Let

$$
\Delta=\sum_{i=1}^{N} \frac{\partial^{2}}{\partial x_{i}^{2}}, \quad \square_{\varepsilon}=\frac{\partial^{2}}{\partial t^{2}}-\varepsilon^{2} \Delta \quad(\varepsilon>0)
$$

and consider the Cauchy problem

$$
\begin{gathered}
\square_{\varepsilon} u_{\varepsilon}=f\left(u_{\varepsilon}\right) \quad \text { in } \mathbf{R}^{N} \times(0, \infty), \\
u_{\varepsilon}(x, 0)=g(x), \quad x \in \mathbf{R}^{N}, \\
\frac{\partial}{\partial t} u_{\varepsilon}(x, 0)=h(x), \quad x \in \mathbf{R}^{N} .
\end{gathered}
$$

Here $f(u)$ is a superlinear function such as $\left(u^{+}\right)^{p}$; more generally we shall assume that $f \geq 0, f \in C^{4}(\mathbf{R})$; there exists a $u_{0} \geq 0$ such that

$$
\begin{aligned}
& f(u)>0, \quad f^{\prime}(u) \geq 0, \quad f^{\prime \prime}(u) \geq 0 \quad \text { if } u \geq u_{0} ; \\
& \left(f(u) / u^{p}\right) \rightarrow 1 \quad \text { if } u \rightarrow \infty, p>1 ; \\
& \underset{u \rightarrow \infty}{\limsup }\left(f^{\prime}(u) / u^{p-1}\right)<p+(p-1) / 2 ; \\
& \liminf _{u \rightarrow \infty}\left(f^{\prime}(u) / u^{p-1}\right)>0 \\
& \left|f^{(j)}(u)\right| \leq C u^{p-j} \quad \text { if } u \geq u_{0}, 2 \leq j \leq 4 .
\end{aligned}
$$

We also assume that

$$
N \leq 3
$$

and

$$
\begin{array}{cl}
g \in C^{5}\left(\mathbf{R}^{N}\right), h \in C^{4}\left(\mathbf{R}^{N}\right) & \text { if } N=2,3 \\
g, h \in C^{4}\left(\mathbf{R}^{1}\right) & \text { if } N=1 .
\end{array}
$$

Received by the editors March 30, 1987 and, in revised form, June 8, 1987.

1980 Mathematics Subject Classification (1985 Revision). Primary 35L70; Secondary 35L05, $35 \mathrm{~L} 67$.

This work is partially supported by National Science Foundation Grants DMS-8420896 and DMS-8501397. 
Sufficient conditions for nonexistence of global solutions of (1.1)-(1.3) are given in [3-7]. In this paper we are interested in the behavior of (blowing-up) solutions $u_{\varepsilon}$ of (1.1)-(1.3) as $\varepsilon \rightarrow 0$. This is naturally related to the behavior of the solutions of the ordinary differential equation

$$
\frac{d^{2} u}{d t^{2}}=f(u) \text { for } t>0
$$

under the Cauchy conditions

$$
u(x, 0)=g(x), \quad u_{t}(x, 0)=h(x) .
$$

For each fixed $x$ the solution of (1.7), (1.8) exists for $0<t<\phi(x)$ where either $\phi(x)=\infty$ or $\phi(x)<\infty$; in the latter case it can be shown (see $\S 2)$ that $u(x, t) \rightarrow \infty$ if $t \rightarrow \phi(x)$, and we say that $u(x, t)$ blows up at time $t=\phi(x)$. The surface $\{t=\phi(x)\}$ is called the blow up surface for $u$.

Caffarelli and Friedman [1] proved that if $N=1$ then there exists a unique classical solution of (1.1)-(1.3) for all $0<t<\phi_{\varepsilon}(x)$ where either $\phi_{\varepsilon}(x) \equiv \infty$ (no blow-up) or else $\phi_{\varepsilon}(x)<\infty$ for all $x \in \mathbf{R}^{1}$ and $\phi_{\varepsilon} \in C^{1},\left|\phi_{\varepsilon}^{\prime}(x)\right|<1 / \varepsilon$; further, $u_{\varepsilon}(x, t) \rightarrow \infty$ if $t \rightarrow \phi_{\varepsilon}(x)$. In [2] they extended these results to $N=2,3$ under some restrictions on the Cauchy data (in addition to (1.6)). We shall recall a slightly simplified version of their result in case $\varepsilon=1$; this will be needed in the sequel.

Introduce the sets

$$
\begin{aligned}
& K^{\varepsilon}\left(x_{0}, t_{0}\right)=\left\{(x, t) ;\left|x-x_{0}\right| \leq \varepsilon\left(t_{0}-t\right), 0 \leq t<t_{0}\right\} \\
& B_{R}\left(x_{0}\right)=\left\{\left|x-x_{0}\right|<R\right\}, \quad B_{R}=B_{R}(0) \\
& K_{R, T}^{\varepsilon}=\bigcup_{x \in B_{R}} K^{\varepsilon}(x, T)
\end{aligned}
$$

We shall assume, in addition to (1.5), (1.6), the following conditions:

(1.9) the solution $w$ of $w^{\prime \prime}(t)=f(w), t>0$ with $w(0)=w^{\prime}(0)=\gamma$ blows up in finite time $T$, where $\gamma>u_{0}, T>0$;

$$
\begin{aligned}
g(x) \geq 2 \gamma, h(x) \geq 2 \gamma & \text { in } B_{R+T}, \\
|\nabla g|+\left|\nabla^{2} g\right|+|\nabla h|<\eta & \text { in } B_{R+T}, n>0 .
\end{aligned}
$$

THEOREM $1.1[2]$. If $\eta$ is sufficiently small, depending on $R, \gamma, T$, then there exists a classical solution $u_{1}(x, t)$ of (1.1)-(1.3) with $\varepsilon=1$ in $K_{R, T}^{1} \cap \Omega$ where $\Omega=\left\{(x, t) ; x \in B_{R+T}, 0<t<\phi_{1}(x)\right\}$, and it satisfies

(i) $0<\phi_{1}(x)<T$,

(ii) $u_{1}(x, t) \rightarrow \infty$ if $t \rightarrow \phi_{1}(x)-0$,

(iii) $\phi_{1} \in C^{1}\left(B_{R+T}\right)$ and $\left|\nabla \phi_{1}(x)\right|<1$. The solution is unique in $K_{R, T}^{1}$ and it belongs to $C^{3,1}$.

The proof of existence of $u_{1}$ begins by constructing a sequence of finite valued solutions $U_{n}$ where $U_{0}=0$ and

$$
\begin{gathered}
\square_{1} U_{n+1}=f\left(U_{n}\right) \quad \text { in } \mathbf{R}^{N} \times(0, \infty), \\
U_{n+1}(x, 0)=g(x), \quad \frac{\partial}{\partial t} U_{n+1}(x, 0)=h(x) \quad\left(x \in \mathbf{R}^{N}\right) .
\end{gathered}
$$

One shows that

$$
U_{n}(x, t) \leq U_{n+1}(x, t) \quad \text { in } K_{R, T}^{1}
$$


and that $U_{n+1}(x, t) \rightarrow u_{1}(x, t)$ as $n \rightarrow \infty$, where $u_{1}$ satisfies the properties asserted in Theorem 1.1.

Consider the case

$$
\phi(0)<\infty
$$

In $\S 2$ we shall prove

LEMMA 1.2. If (1.13) holds then there exists an $R^{\prime}>0$ such that

$$
\begin{gathered}
0<\phi(x)<\infty \quad \text { if } x \in B_{R^{\prime}}, \\
\phi \in C^{1}\left(B_{R^{\prime}}\right) .
\end{gathered}
$$

Actually $\phi$ belongs to $C^{4}\left(B_{R^{\prime}}\right)$, but this fact will not be needed.

In $\S 3$ we shall prove

LEMMA 1.3. Fix any $T$ such that $\phi(0)<T<\infty$. Then there exist $R>$ $0, C>0$ and $\varepsilon_{0}>0$ such that if $0<\varepsilon<\varepsilon_{0}$ then there exists a unique solution $u_{\varepsilon}$ of (1.1)-(1.3) in $K_{R, T}^{\varepsilon} \cap \Omega_{R, T}^{\varepsilon}$ where $\Omega_{R, T}^{\varepsilon}=\left\{(x, t) ; x \in B_{R+T}, 0<t<\phi_{\varepsilon}(x)\right\}$ and

(i) $0<\phi_{\varepsilon}(x)<T$,

(ii) $u_{\varepsilon}(x, t) \rightarrow \infty$ if $t \rightarrow \phi_{\varepsilon}(x)-0, x \in B_{R}$,

(iii) $\phi_{\varepsilon} \in C^{1}\left(B_{R+T}\right)$ and $\left|\nabla \phi_{\varepsilon}(x)\right| \leq C$; the solution belongs to $C^{3,1}$.

We can now state the main results of this paper in case $\phi(0)<\infty$.

THEOREM 1.4. If (1.4)-(1.6) hold and $\phi(0)<\infty$ then there exist positive contants $R, C$ such that, for all $\varepsilon$ sufficiently small,

$$
\begin{aligned}
& \sup _{B_{R}}\left|\phi_{\varepsilon}(x)-\phi(x)\right| \leq C \varepsilon^{2}, \\
& \sup _{B_{R}}\left|\nabla\left(\phi_{\varepsilon}(x)-\phi(x)\right)\right| C \varepsilon^{2} .
\end{aligned}
$$

Theorem 1.4 will be proved in $\S \S 4-6$.

Observe that Lemma 1.2 implies that the set $D=\left\{x \in \mathbf{R}^{N}, \phi(x)<\infty\right\}$ if open, and Theorem 1.4 implies that the solution $u_{\varepsilon}$ exists for $0<t<\phi_{\varepsilon}(x)$ and $x$ in any compact subset $D_{0}$ of $D$; further

$$
\begin{gathered}
\left|\phi_{\varepsilon}(x)-\phi(x)\right| \leq C \varepsilon^{2} \quad \forall x \in D_{0}, \\
\left|\nabla\left(\phi_{\varepsilon}(x)-\phi(x)\right)\right| \leq C \varepsilon^{2} \quad \forall x \in D_{0} .
\end{gathered}
$$

In $\S 7$ we shall consider the case $\phi(0)=\infty$ and derive growth rates for $\phi_{\varepsilon}(0)$ as $\varepsilon \rightarrow 0$.

2. The equation $u^{\prime \prime}=f(u)$. Throughout $\S \S 2-6$ we assume that (1.13) holds.

Set

$$
F(u)=\int_{0}^{u} f(s) d s .
$$

From (1.7), (1.8) we obtain

$$
u_{t}^{2}-h^{2}(x)=2[F(u)-F(g(x))] \quad \text { if } t<\phi(x)
$$


and then also

$$
\phi(x)=\int_{g(x)}^{\infty} \frac{d u}{\left[2 F(u)-2 F(g(x))+h^{2}(x)\right]^{1 / 2}}
$$

provided, say, $g(x)>u_{0}$ (so that the denominator in the integrand is well defined).

Set

$$
T(\gamma, \delta)=\int_{\gamma}^{\infty} \frac{d u}{\left[2 F(u)-2 F(\gamma)+\delta^{2}\right]^{1 / 2}}
$$

for $\gamma>u_{0}, \delta>0$. Then $T(\gamma, \delta)$ is the blow-up time of (1.7) subject to $u(0)=$ $\gamma, u^{\prime}(0)=\delta$. Using (1.4) we can easily show that $u^{\prime}(t)>0, u^{\prime \prime}(t)>0$ if $t>0$ and then, from the differential equation for $\partial u / \partial \gamma, \partial u / \partial \gamma$ remains positive for all $0<t<T(\gamma, \delta)$. It follows that $\partial T / \partial \gamma \leq 0$. Since

$$
\frac{\partial T}{\partial \gamma}=-\frac{1}{\delta}+f(\gamma) \int_{\gamma}^{\infty} \frac{d u}{\left[2 F(u)-2 F(\gamma)+\delta^{2}\right]^{3 / 2}}
$$

we deduce that

$$
|\partial T / \partial \gamma| \leq 1 / \delta
$$

Next

$$
\frac{\partial T}{\partial \delta}=-\delta \int_{\gamma}^{\infty} \frac{d u}{\left[2 F(u)-2 F(\gamma)+\delta^{2}\right]^{3 / 2}}
$$

so that

$$
|\partial T / \partial \delta| \leq T / \delta
$$

The assumption $\phi(0)<\infty$ implies that

$$
u(0, t) \rightarrow \infty \text { if } t \rightarrow \phi(0) .
$$

Indeed, if $u\left(0, t_{n}\right)$ remains bounded for a sequence $t_{n} \rightarrow \phi(0)$, then, by $(2.1)$, also $u_{t}\left(0, t_{n}\right)$ remains bounded. But then the solution $u(0, t)$ of $u_{t t}=f(u)$ can be extended to $t_{n}<t<t_{n}+\delta$ with $\delta$ positive and independent of $n$, which is a contradiction if $n$ is large enough.

From (2.6) and (2.1) we get

$$
u_{t}(0, t) \rightarrow \infty \text { if } t \rightarrow \phi(0) .
$$

Consequently, for any $\gamma>u_{0}$ there exists a $t_{0} \in(0, \phi(0))$ such that $u\left(0, t_{0}\right)>$ $\gamma, u_{t}\left(0, t_{0}\right)>\gamma$ and, by continuity

$$
u\left(x, t_{0}\right)>\gamma, \quad u_{t}\left(x, t_{0}\right)>\gamma \quad \text { if } x \in B_{R_{0}}
$$

for some $R_{0}>0$. Using (1.4) we easily deduce that $u(x, t)$ blows up in finite time $\phi(x)$ for any $x \in B_{R_{0}}$. Further, analogously to (2.2), we have

$$
\phi(x)=t_{0}+T\left(u\left(x, t_{0}\right), u_{t}\left(x, t_{0}\right)\right), \quad x \in B_{R_{0}} .
$$

Since $u\left(x, t_{0}\right)$ and $u_{t}\left(x, t_{0}\right)$ vary smoothly with $x$ and since (2.4), (2.5) hold, we conclude: 
LEMMA 2.1. $\phi \in C^{1}\left(B_{R_{0}}\right)$.

Set

$$
\Omega_{\rho}=\left\{(x, t) ; x \in B_{\rho}, 0 \leq t<\phi(x), \rho<R_{0}\right\} .
$$

LEMMA 2.2. For any $0<R<R_{0}$ there exist positive constants $C$ and $c$ such that

$$
\left|D^{\alpha} u(x, t)\right| \leq C(\phi(x)-t)^{-(p q+|\alpha|-2)} \text { in } \Omega_{R}
$$

where $q=2 /(p-1), 0 \leq|\alpha| \leq 2$, and

$$
c(\phi(x)-t)^{-(p q+j-2)} \leq D_{t}^{j} u(x, t) \leq C(\phi(x)-t)^{-(p q+j-2)} \quad \text { in } \Omega_{R}
$$

for $0 \leq j \leq 3$.

ProOF. From (2.1), by integration,

$$
\int_{u(x, t)}^{\infty} \frac{d u}{\left[h^{2}(x)+2 F(u)-2 F(g(x))\right]^{1 / 2}}=\phi(x)-t .
$$

Since $F(u) \sim u^{p+1} /(p+1)$ as $u \rightarrow \infty$, the estimate (2.11) for $u$ readily follows. Next using (2.1) we can establish (2.11) for $D_{t} u$, and using (1.7) we can further establish (2.11) for $j=2$ and then (from $u_{t t t}=f^{\prime}(u) u_{t}$ ) for $j=3$.

To prove (2.10) we introduce (cf. [2]) the functions

$$
\begin{array}{ll}
J_{1}=C_{1} u_{t} \pm D^{\alpha} u, & |\alpha|=1, \\
J_{2}=C_{2} u_{t t} \pm D^{\alpha} u, & |\alpha|=2,
\end{array}
$$

with $C_{1}, C_{2}$ positive constants. For any $x_{0} \in B_{R}$ we can choose $\delta>0$ and $t_{1} \in$ $\left(0, \phi\left(x_{0}\right)\right)$ such that $u\left(x, t_{1}\right)>u_{0}$ and $D_{t}^{j} u\left(x, t_{1}\right)>1$ if $x \in B_{\delta}\left(x_{0}\right)(0 \leq j \leq 3)$. Hence, if $C_{1}$ is large enough then $J_{1}\left(x, t_{1}\right)>0$ and $J_{1, t}\left(x, t_{1}\right)>0$ for $x \in B_{\delta}\left(x_{0}\right)$. Since

$$
d^{2} J_{1} / d t^{2}=f^{\prime}(u) J_{1},
$$

we can easily deduce by a continuity argument that $J_{1}(x, t)$ remains positive for $t_{1}<t<\phi(x)$, if $x \in B_{\delta}\left(x_{0}\right)$.

Next we choose $C_{2}$ such that $J_{2}\left(x, t_{1}\right)>0$ and $J_{2, t}\left(x, t_{1}\right)>0$ for $x \in B_{\delta}\left(x_{0}\right)$. We have

$$
\frac{d^{2} J_{2}}{d t^{2}}=f^{\prime}(u) J_{2}+f^{\prime \prime}(u)\left(C_{2} u_{t}^{2} \pm D^{\beta_{1}} u D^{\beta_{2}} u\right)
$$

where $\beta_{1}+\beta_{2}=\alpha$. Since $J_{1}>0$, if $C_{2}$ is large enough then the coefficient of $f^{\prime \prime}(u)$ is positive. Hence, by a continuity argument, $J_{2}(x, t)>0$ if $x \in B_{\delta}\left(x_{0}\right), t_{1}<t<$ $\phi(x)$. Combining the positivity of $J_{1}, J_{2}$ with (2.11), the estimate (2.10) follows.

3. Proof of Lemma 1.3. In the sequence we shall need an integral representation for solutions of the inhomogeneous wave equation. The formula has a different form depending on the space dimension $N$. We shall consider only the case $N=3$; the cases $N=1,2$ can be treated in a similar way.

For $N=3$ we have

$$
\begin{aligned}
w(x, t)= & \frac{t}{4 \pi} \int_{|\xi|=1} w_{1}(x+\varepsilon t \xi) d \omega_{\xi}+\frac{\partial}{\partial t} \frac{t}{4 \pi} \int_{|\xi|=1} w_{0}(x+\varepsilon t \xi) d \omega_{\xi} \\
& +\frac{1}{4 \pi} \int_{0}^{t}(t-s) d s \int_{|\eta|=1} h(x+\varepsilon(t-s) \eta, s) d \omega_{\eta}
\end{aligned}
$$


where

$$
w_{0}(y)=w(y, 0), \quad w_{1}(y)=w_{t}(y, 0), \quad h(y, t)=\square_{\varepsilon} w(y, t) .
$$

For any $R_{1}>0,0<\varepsilon<1$ we can construct a solution $u_{\varepsilon}$ of (1.1)-(1.3) in $K_{R_{1}, \sigma_{1}}^{\varepsilon}$ provided $\sigma_{1}$ is sufficiently small, independently of $\varepsilon$. In fact, define an operator $S$ by

$$
(S w)(x, t)=G(x, t)+\frac{1}{4 \pi} \int_{0}^{t}(t-s) d s \int_{|\eta|=1} f(w(x+\varepsilon(t-s) \eta, s)) d \omega_{\eta},
$$

where

$$
G(x, t)=\frac{t}{4 \pi} \int_{|\xi|=1} h(x+\varepsilon t \xi) d w_{\xi}+\frac{\partial}{\partial t} \frac{t}{4 \pi} \int_{|\xi|=1} g(x+\varepsilon t \xi) d \omega_{\xi} .
$$

The domain of $S$ is taken to be

$$
X_{\sigma_{1, M_{1}}}=\left\{w \in C^{0}\left(K_{R_{1}, \sigma_{1}}^{\varepsilon}\right) ; \sup |w| \leq M_{1}\right\}
$$

where

$$
M_{1}=1+\sup _{K_{R_{1}, T}^{\epsilon}}|G| .
$$

Then, if $\sigma_{1}$ is sufficiently small, $S$ maps $X_{\sigma_{1}, M_{1}}$ into itself and it is a contraction. It follows that $S$ has a fixed point, which is clearly a solution $u_{\varepsilon}$ of (1.i)-(1.3). If $\tilde{u}_{\varepsilon}$ is another solution in $K_{R_{1}, \sigma_{2}}$ then we easily deduce that

$$
\left\|S \tilde{u}_{\varepsilon}-S u_{\varepsilon}\right\|<\frac{1}{2}\left\|\tilde{u}_{\varepsilon}-u_{\varepsilon}\right\|
$$

where the norm is the supremum norm in $K_{R_{1}, \sigma}$ with $\sigma$ small enough, depending on $\left\|\tilde{u}_{\varepsilon}\right\|$. It follows that $\tilde{u}_{\varepsilon}=u_{\varepsilon}$ if $0<t<\sigma$, and by a step-by-step argument, also if $0<t<\min \left(\sigma_{1}, \sigma_{2}\right)$.

Since $\square_{\varepsilon}\left(D U_{\varepsilon}\right)=f^{\prime}\left(u_{\varepsilon}\right)\left(D u_{\varepsilon}\right)$, we can apply (3.1) to $D u_{\varepsilon}$ and, by estimating successively the right-hand side, we find that

$$
\left|D u_{\varepsilon}\right| \leq M_{1}^{\prime} \text { in } K_{R_{1}, \sigma_{1}}^{\varepsilon}
$$

provided $\sigma_{1}$ is small enough; $\sigma_{1}$ and $M_{1}^{\prime}$ are independent of $\varepsilon$. Similarly

$$
\left|D^{\alpha} u_{\varepsilon}\right| \leq M_{1}^{\prime} \text { in } K_{R_{1}, \sigma_{1}}^{\varepsilon},|\alpha| \leq 3,
$$

with another constant $M_{1}^{\prime}$.

From (3.4) it follows that any sequence $\varepsilon \rightarrow 0$ has a subsequence such that

(3.5) $D^{\alpha} u_{\varepsilon} \rightarrow D^{\alpha} v$ in $\left(L^{\infty}\left(K_{R_{1}, \sigma_{1}}^{\varepsilon}\right)\right)^{*}$ weakly; $0 \leq|\alpha| \leq 3$, and, therefore

(3.6) $D^{\alpha} u_{\varepsilon} \rightarrow D^{\alpha} v$ uniformly in compact subsets of $\bigcap_{\varepsilon^{\prime}} K_{R_{1}, \sigma_{1}}^{\varepsilon^{\prime}} ; 0 \leq|\alpha| \leq 2$. Hence

$$
v(x, t)=u(x, t)
$$

where $u$ is the solution of (1.7), (1.8) and (3.5), (3.6) hold for all $\varepsilon \rightarrow 0$.

We wish to extend the solution $u_{\varepsilon}$ beyond $t=\sigma_{1}$. To do this we repeat the previous proof, considering $S$ in the space

$$
X_{\sigma_{2}, M_{2}}=\left\{w \in C^{0}\left(K_{R_{2}, \sigma_{2}}^{\varepsilon}\right), w \equiv u_{\varepsilon} \text { in } K_{R_{2}, \sigma_{2}}^{\varepsilon} \cap\left\{t<\sigma_{1}\right\}, \sup |w| \leq M_{1}\right\}
$$


for any $R_{2}<R_{1}, M_{2}=M_{1}+1$ and some $\sigma_{2}>\sigma_{1}$. Then $S$ is a contraction if $\sigma_{2}-\sigma_{1}$ is sufficiently small, depending on $M_{2}$. As before we can establish (3.5)-(3.7) in $K_{R_{2}, \sigma_{2}}^{\varepsilon}$.

We can carry out the above procedure with $\sigma_{3}, R_{3}, M_{3}, \sigma_{4}, R_{4}, M_{4}$, etc.; however, the numbers $\sigma_{j+1}-\sigma_{j}$ are decreasing since the $M_{j}$ are increasing. Let

$$
\tilde{\sigma}<\inf _{B_{R_{1}}} \phi(x), \quad 0<\tilde{R}<R_{1}, R_{1} \text { small. }
$$

We claim that the above procedure yields, in a finite number $j_{0}$ of steps $\left(j_{0}\right.$ independent of $\varepsilon$ ) a solution $u_{\varepsilon}$ in $K_{\tilde{R}, \tilde{\sigma}}$. Indeed, from (3.8) we have that

$$
\left|D^{\alpha} u(x, t)\right| \leq C \quad \text { in } B_{R_{1}} \times[0, \tilde{\sigma}](|\alpha| \leq 3) .
$$

Hence, in view of (3.5)-(3.7) we may repeat the previous construction of $u_{\varepsilon}$ but with the following modifications: at each step $j$ we must take $\varepsilon \leq \varepsilon_{j}$ so that for $|\alpha| \leq 2$ we have $\left|D^{\alpha} u_{\varepsilon}\right|<C+1$ in $K_{R_{j}, \sigma_{j}}$. Hence we obtain the bound $C+2$ instead of $M_{j}$ for $\left|D^{\alpha} u_{\varepsilon}\right|$ in $K_{R_{j+1}, \sigma_{j+1}}(|\alpha| \leq 2)$. Next, by Gronwall's inequality we can derive a bound $M_{j+1}$, independent of $\varepsilon$, for $\left|D^{\alpha} u_{\varepsilon}\right|$ in $K_{R_{j+1}, \sigma_{j+1}}$ where $|\alpha|=3$. We have $\varepsilon_{1}>\varepsilon_{2}>\cdots$. However, the differences $\sigma_{j+1}-\sigma_{j}$ remain uniformly positive independently of the choice of the $R_{j}$; say $\sigma_{j+1}-\sigma_{j} \geq \delta>0\left(\varepsilon_{j}\right.$ depends on $R_{j}$ ). Choosing $j_{0}=[\tilde{\sigma} / \delta]+1$, and $R_{j}=R_{1}-\left(R_{1}-\tilde{R}\right) / j_{0}$, we obtain the desired solution $u_{\varepsilon}$ in $K_{\tilde{R}, \tilde{\sigma}}^{\varepsilon}$. Further,

(3.9) $D^{\alpha} u_{\varepsilon} \rightarrow D^{\alpha} u$ uniformly in compact subsets of $B_{\tilde{R}} \times[0, \tilde{\sigma}], 0 \leq|\alpha| \leq 2$.

Choosing $R_{1}$ sufficiently small, we can take $\tilde{\sigma}$ sufficiently close to $\phi(0)$. Hence in view of $(2.8),(3.9)$ we have

$$
u_{\varepsilon}\left(x, t_{0}\right)>2 \gamma, u_{\varepsilon, t}\left(x, t_{0}\right)>2 \gamma \quad \text { if } x \in B_{2 R},
$$

provided $2 R<\tilde{R}$, where $\gamma>u_{0}$ and $t_{0}$ is some point in $(0, \tilde{\sigma})$.

We now introduce the scaled functions

$$
U_{\varepsilon}(x, t)=u_{\varepsilon}(\varepsilon x, t) \text { for } t \geq t_{0} .
$$

Then $\square_{1} U_{\varepsilon}=f\left(U_{\varepsilon}\right)$. Setting $g_{\varepsilon}(x)=U_{\varepsilon}\left(x, t_{0}\right), h_{\varepsilon}(x)=U_{\varepsilon, t}\left(x, t_{0}\right)$, we have

$$
\left|\nabla g_{\varepsilon}\right|+\left|\nabla h_{\varepsilon}\right| \leq C \varepsilon, \quad\left|\nabla^{2} g_{\varepsilon}\right| \leq C \varepsilon^{2} .
$$

Using (3.10), (3.11) we can now apply the proof of Theorem 1.4 (as given in [2]) in order to establish the existence of a unique solution $U_{\varepsilon}$ in $K_{(R / \varepsilon), T}^{1} \cap\{t \geq$ $\left.t_{0}\right\} \cap\left\{t<\tilde{\phi}_{\varepsilon}(x)\right\}$ for all $\varepsilon$ small enough, and the estimate

$$
U_{\varepsilon, t} \geq\left(c_{0} / \varepsilon\right)\left|\nabla_{x} U_{\varepsilon}\right|, \quad c_{0}>0:
$$

the function $U_{\varepsilon}(x / \varepsilon, t)$ is then the unique extension of the solution $u_{\varepsilon}$ to $\left\{t<\phi_{\varepsilon}(x)\right\}$, where $\phi_{\varepsilon}(x)=\tilde{\phi}(\varepsilon x)$. It will be shown below that

$$
\phi_{\varepsilon}(x)<T \text {. }
$$

Then, the assertion (ii) of Lemma 1.3 follows (from the proof of Theorem 1.4 for $\left.U_{\varepsilon}\right)$, and $\phi_{\varepsilon} \in C^{1}$. Further, from (3.12) we deduce that $\left|\nabla \phi_{\varepsilon}\right| \leq 1 / c_{0}$ and thus Lemma 1.3 follows. 
To prove (3.13) let $W_{\delta}(t)$ ( $\delta$ positive and small) be the solution of

$$
\begin{gathered}
W_{\delta}^{\prime \prime}=f\left(W_{\delta}\right) \quad \text { if } t>t_{0} \\
W_{\delta}\left(t_{0}\right)=u\left(0, t_{0}\right)-2 \delta \\
W_{\delta}^{\prime}\left(t_{0}\right)=u_{t}\left(0, t_{0}\right)-2 \delta
\end{gathered}
$$

We shall compare $W_{\delta}$ with $U_{\varepsilon}$ in $K_{(R / \varepsilon), T}$. By (3.9), if $R$ is small enough, depending on $\delta$, then

$$
\begin{gathered}
W_{\delta}\left(t_{0}\right) \leq U_{\varepsilon}\left(x, t_{0}\right)-\delta \\
W_{\delta}^{\prime}\left(t_{0}\right) \leq U_{\varepsilon, t}\left(x, t_{0}\right)-\delta \quad \text { in } B_{(R / \varepsilon)+T}
\end{gathered}
$$

provided $\varepsilon$ is sufficiently small. Also,

$$
\left|\nabla U_{\varepsilon}\left(x, t_{0}\right)\right| \leq C \varepsilon<\delta \quad \text { if } \varepsilon<\delta / C .
$$

Hence, by a comparison argument based on the representation (3.1), (3.2) (cf. the proof of Lemma 2.3 in [2]), it follows that

$$
W_{\delta}(t) \leq U_{\varepsilon}(x, t) \quad \text { in } K_{(R / \varepsilon), T},
$$

and thus $\phi_{\varepsilon}(x)<T_{\delta}$ where $T_{\delta}$ is the blow-up time for $W_{\delta}$. By the results of $\S 2$ (cf. (2.4), (2.5)), $\left|T_{\delta}-\phi(x)\right|<C_{1} \delta$. Consequently

$$
\phi_{\varepsilon}(x) \leq \phi(x)+C_{1} \delta
$$

and (3.13) follows.

REMARK 3.1 . If we replace $-\delta$ by $+\delta$ in (3.14) then the previous argument yields the estimate

$$
\phi_{\varepsilon}(x) \geq \phi(x)-C_{1} \delta
$$

4. Estimating $u_{\varepsilon}-u$.

LEMMA 4.1. If $R$ is sufficiently small then for any compact subset $D_{0}$ of

$$
\Omega_{R} \equiv\left\{(x, t) ; x \in B_{R}, 0 \leq t<\phi(x)\right\}
$$

there exists a constant $C$ such that

$$
\begin{aligned}
& \left|u_{\varepsilon}-u\right| \leq C \varepsilon^{2} \quad \text { in } D_{0} \\
& \left|u_{\varepsilon, t}-u_{t}\right| \leq C \varepsilon^{2} \quad \text { in } D_{0}
\end{aligned}
$$

if $\varepsilon$ is sufficiently small.

PROOF. By the estimates of $\S 3$ we deduce that if $\rho$ is sufficiently small then

$$
\left|D^{\alpha} u_{\varepsilon}\right| \leq C \text { for } 0 \leq|\alpha| \leq 3
$$

provided $(x, t) \in D_{0}$ and $|x|<\rho$. Similarly, for any $x_{0} \in B_{R}$ the estimate (4.3) holds on $\left\{(x, t) \in D_{0}, x \in B_{\rho}\left(x_{0}\right)\right\}$, where $C$ and $\rho$ can be taken independently of $x_{0}$. It follows that (4.3) holds in $D_{0}$.

We can then write

$$
\frac{\partial^{2}}{\partial t^{2}} u_{\varepsilon}=f\left(u_{\varepsilon}\right)+h_{\varepsilon}, \quad\left|h_{\varepsilon}\right|=\left|\varepsilon^{2} \Delta u_{\varepsilon}\right| \leq C \varepsilon^{2} .
$$


Representing $u$ in the form

$$
u(x, t)=g(x)+t h(x)+\int_{0}^{t}(t-\tau) f(u(x, \tau)) d \tau
$$

and, similarly,

$$
\begin{aligned}
u_{\varepsilon}(x, t)= & g(x)+t h(x)+\int_{0}^{t}(t-\tau) f\left(u_{\varepsilon}(x, \tau)\right) d \tau \\
& +\int_{0}^{t}(t-\tau) h_{\varepsilon}(x, \tau) d \tau
\end{aligned}
$$

and taking the difference, we get

$$
\left|u_{\varepsilon}(x, t)-u(x, t)\right| \leq C \int_{0}^{t}\left|u_{\varepsilon}(x, \tau)-u(x, \tau)\right|+C \varepsilon^{2}
$$

provided $D_{0}$ is taken to be a subgraph in the $t$-direction, and (4.1) follows.

Similarly

and

$$
\begin{gathered}
\frac{\partial^{2}}{\partial t^{2}} u_{\varepsilon, t}=f^{\prime}\left(u_{\varepsilon}\right) u_{\varepsilon, t}+h_{1}, \quad\left|h_{1}\right|=\left|\varepsilon^{2} \Delta u_{\varepsilon, t}\right| \leq C \varepsilon^{2} \\
\frac{\partial^{2}}{\partial t^{2}} u_{t}=f^{\prime}(u) u_{t}
\end{gathered}
$$

$$
\begin{gathered}
u_{\varepsilon, t}(x, 0)=h(x)=u_{t}(x, 0), \\
u_{\varepsilon, t t}(x, 0)=\varepsilon^{2} \Delta g+f(g)=u_{t t}(x, 0)+\varepsilon^{2} \Delta g
\end{gathered}
$$

and the previous argument coupled with the estimate (4.1) yields the assertion (4.2).

5. Estimating $\phi_{\varepsilon}-\phi$ and estimating $D^{\alpha}\left(u_{\varepsilon}-u\right)$ near $\{t=\phi\}$.

LEMMA 5.1. If $R$ is sufficiently small then there exists a constant $C$ such that

$$
\sup _{B_{R}}\left|\phi_{\varepsilon}(x)-\phi(x)\right| \leq C \varepsilon
$$

for all $\varepsilon$ sufficiently small.

PROOF. We re-examine the proof of (3.17), (3.18). It is easy to see that (3.15) holds with $\delta=A \varepsilon$ provided $A$ is a sufficiently large positive number. Recalling also (3.16), we deduce as before that (3.17) holds if $\delta=C \varepsilon$ and $\varepsilon$ is sufficiently small. The proof of (3.18) with $\delta=C \varepsilon$ is similar.

In the sequel we shall need some estimates on $D^{\alpha} u_{\varepsilon}$ and $D^{\alpha}\left(u_{\varepsilon}-u\right)$ near the blow-up surface. We begin with

LEMMA 5.2. There exist $t_{1} \in(0, \phi(0))$ and $R>0$ such that, for all $\varepsilon$ sufficiently small,

$$
\begin{gathered}
c\left(\phi_{\varepsilon}(x)-t\right)^{(p q+j-2)} \leq D_{t}^{j} u_{\varepsilon}(x, t) \leq C\left(\phi_{\varepsilon}(x)-t\right)^{-(p q+j-2)} \quad(0 \leq j \leq 2) \\
\left|D^{\alpha} u_{\varepsilon}(x, t)\right| \leq C\left(\phi_{\varepsilon}(x)-t\right)^{-(p q+|\alpha|-2)} \quad(0 \leq|\alpha| \leq 2)
\end{gathered}
$$

for $x \in B_{R}, t_{1}<t<\phi_{\varepsilon}(x)$, where $c, C$ are positive constants, and $q=2 /(p-1)$. 
PROOF. To prove (5.2) we work with $U_{\varepsilon}(x, t)=u_{\varepsilon}(\varepsilon x, t)$ and establish for $D_{t}^{j} U_{\varepsilon}$ estimates (as in (5.2) with $\phi_{\varepsilon}(x)$ replaced by $\phi_{\varepsilon}(\varepsilon x)$ ) by the method of [2]. Since $D_{t}^{j} u_{\varepsilon}(x, t)=D_{t}^{j} U_{\varepsilon}(x / \varepsilon, t)$, the inequalities in (5.2) follow.

Consider next

$$
\begin{array}{cc}
J_{1}^{\varepsilon}=C_{1} u_{\varepsilon, t} \pm D_{x}^{\alpha} u_{\varepsilon} & (|\alpha|=1), \\
J_{2}^{\varepsilon}=C_{2} u_{\varepsilon, t t} \pm D_{x}^{\alpha} u_{\varepsilon} & (|\alpha|=2) .
\end{array}
$$

If $C_{1}, C_{2}$ are positive and sufficiently large, then

$$
J_{i}^{0}\left(x, t_{1}\right)>0, J_{i, t}^{0}\left(x, t_{1}\right)>0 \text { if } x \in B_{R},
$$

where $J_{i}^{0}$ is $J_{i}^{\varepsilon}$ with $u_{\varepsilon}$ replaced by $u$. Consequently, by (3.9),

$$
J_{i}^{\varepsilon}\left(x, t_{1}\right)>0, J_{i, t}^{\varepsilon}\left(x, t_{1}\right)>0 \text { if } x \in B_{R}
$$

provided $\varepsilon$ is sufficiently small. We can now proceed by a comparison argument as in [2] (cf. also the proof of Lemma 2.2) to show that if $\varepsilon$ is small enough (so that also $\left.\left|\nabla_{x} J_{1}^{\varepsilon}\left(\varepsilon x, t_{1}\right)\right|<\varepsilon J_{1, t}^{\varepsilon}\left(\varepsilon x, t_{1}\right), \varepsilon x \in B_{R}\right)$ then $J_{1}^{\varepsilon}(x, t)>0$ in the set $\left(\bigcup_{x_{0} \in B_{r}} K^{\varepsilon}\left(x_{0}, \phi_{\varepsilon}\left(x_{0}\right)\right)\right) \cap\left\{t>t_{1}\right\}$. This yields the assertion (5.3) for $|\alpha|=1$ (with a different $R$ ). Using this information we can next establish by comparison that $J_{2}^{\varepsilon}(x, t)>0$ in the same domain as before provided $C_{2}$ is suitably chosen, and (5.3) thus follows for $|\alpha|=2$.

By (5.1) we know that

$$
\left|\phi_{\varepsilon}(x)-\phi(x)\right| \leq C_{0} \varepsilon \quad \text { if } x \in B_{R} .
$$

We shall choose a constant $M$ such that $M>2 C_{0}$. Then

$$
\tilde{c} \leq \frac{\phi_{\varepsilon}(x)-t}{\phi(x)-t}<\frac{1}{\tilde{c}} \quad \text { if } x \in B_{R}, 0<t<\phi(x)-M \varepsilon
$$

where $\tilde{c}$ is a positive constant independent of $\varepsilon$.

LEMMA 5.3. The following estimates hold for $t_{1}<t<\phi(x)-M \varepsilon, x \in B_{R}$ :

$$
\left|D^{\alpha}\left(u_{\varepsilon}-u\right)(x, t)\right| \leq C \varepsilon^{2}(\phi(x)-t)^{-(p q+|\alpha|-2)} \quad(0 \leq|\alpha| \leq 2)
$$

where $C$ is a positive constant independent of $\varepsilon$.

PROOF. We proceed as in Lemma 4.1 but use the estimates of Lemma 5.2. From the integral representation of $u_{\varepsilon}$ and $u$ in (4.6) and (4.5) we obtain, by taking the difference,

$$
\left|u_{\varepsilon}(x, t)-u(x, t)\right| \leq C \varepsilon^{2} \int_{t_{1}}^{t} \int_{t_{1}}^{\tilde{t}}\left|\Delta u_{\varepsilon}\right|+\int_{t_{1}}^{t} \int_{t_{1}}^{\tilde{t}} f^{\prime}(\tilde{u})\left|u_{\varepsilon}-u\right|+C \varepsilon^{2}
$$

where, in each integral, both integrations are in $t$, and $\tilde{u}$ lies between $u$ and $u_{\varepsilon}$. Setting $\lambda=\phi(x)-t$ ( $x$ fixed) and using Lemma 5.2, we obtain

$$
\begin{aligned}
\left|u_{\varepsilon}\right| & \leq C \varepsilon^{2} \int_{t_{*}}^{\lambda} \int_{t_{*}}^{\tilde{\lambda}} \frac{1}{\tilde{\lambda}^{p q}}+C \int_{t_{*}}^{\lambda} \int_{t_{*}}^{\tilde{\lambda}} \frac{\left|u_{\varepsilon}-u\right|}{\tilde{\lambda}(p q-2)(p-1)}+C \varepsilon^{2} \\
& \leq \frac{C \varepsilon^{2}}{\lambda^{p q-2}}+C \int_{t_{*}}^{\lambda} \int_{t_{*}}^{\tilde{\lambda}} \frac{\left|u_{\varepsilon}-u\right|}{\tilde{\lambda}^{2}} \\
& \leq \frac{C \varepsilon^{2}}{\lambda^{p q-2}}+\frac{C}{\lambda} \int_{t_{*}}^{\lambda}\left|u_{\varepsilon}-u\right| \quad\left(\phi(x)-t_{1}=t_{*}\right) .
\end{aligned}
$$


Hence the function

$$
z=\int_{t_{*}}^{\lambda}\left|u_{\varepsilon}-u\right|
$$

satisfies

$$
z^{\prime}-C z / \lambda \leq C \varepsilon^{2} / \lambda^{p q-2}, \quad z\left(t_{*}\right)=0,
$$

from which we deduce that $z \leq C \varepsilon^{2} / \lambda^{p q-3}$. Hence

$$
\left|u_{\varepsilon}-u\right| \leq C \varepsilon^{2} /(\phi(x)-t)^{p q-2} .
$$

This establishes (5.6) for $|\alpha|=0$.

To consider the case $|\alpha|=1$ we first take $D^{\alpha}=D_{t}$. By differentiating the integral representation of $u_{\varepsilon}$ and $u$ with respect to $t$ and taking the difference, we get

$$
\left|u_{\varepsilon, t}-u_{t}\right| \leq c \varepsilon \int_{t_{1}}^{t}\left|\Delta u_{\varepsilon}\right|+\int_{t_{1}}^{t} f^{\prime}(\tilde{u})\left|u_{\varepsilon}-u\right| .
$$

Using (5.7) and Lemma 5.2, we easily estimate the right-hand side, thereby deriving (5.6).

To estimate $D_{x}^{\alpha}\left(u_{\varepsilon}-u\right)$ for $|\alpha|=1$ we apply $D_{x}^{\alpha}$ to the integral representation of $u_{\varepsilon}-u$ and obtain

$$
\left|D_{x}^{\alpha}\left(u_{\varepsilon}-u\right)\right| \leq C \varepsilon^{2} \iint\left|D_{x}^{\alpha} \Delta u_{\varepsilon}\right|+\iint\left|f^{\prime}\left(u_{\varepsilon}\right) D_{x}^{\alpha} u_{\varepsilon}-f^{\prime}(u) D_{x}^{\alpha} u\right| .
$$

Estimating

$$
\left|\left[f^{\prime}\left(u_{\varepsilon}\right)-f^{\prime}(u)\right] D_{x}^{\alpha} u_{\varepsilon}\right|
$$

by (5.7) and Lemma 5.2 we find that

$$
\left|D_{x}^{\alpha}\left(u_{\varepsilon}-u\right)\right| \leq \frac{C \varepsilon^{2}}{\lambda^{p q-1}}+C \int_{t_{*}}^{\lambda} \int_{t_{*}}^{\tilde{\lambda}} \frac{\left|D_{x}^{\alpha}\left(u_{\varepsilon}-u\right)\right|}{\tilde{\lambda}(p q-2)(p-1)} .
$$

We can now proceed as before to establish (5.6) (with $|\alpha|=1$ ). Finally, the proof of (5.6) for $|\alpha|=2$ is similar; we argue separately in the cases $D_{t}^{2}, D_{t} D_{x}^{\alpha}(|\alpha|=1)$ and $D_{x}^{\alpha}(|\alpha|=2)$.

Using Lemmas 5.2 and 5.3 we shall now complete the proof of (1.16).

LEMMA 5.4. If $R$ is sufficiently small then there exists a constant $C$ such that

$$
\sup _{B_{R}}\left|\phi_{\varepsilon}(x)-\phi(x)\right| \leq C \varepsilon^{2}
$$

for all $\varepsilon$ sufficiently small.

PROOF. We repeat the proof of Lemma 5.1 choosing, in the comparison argument (3.14),

$$
t_{0}=\phi(0)-3 M \varepsilon
$$

and working in the cone $K$ with base $B_{5 M \varepsilon^{2}}(0)$ on $t=t_{0}$ and vertex $(0, \phi(0)+2 M \varepsilon)$. Set $d=3 M \varepsilon$. By Lemmas 5.2 and 5.3,

$$
\begin{array}{ll}
\gamma_{\varepsilon} \equiv u\left(0, t_{0}\right)+C_{0} \varepsilon^{2} / d^{p q-1} \geq u_{\varepsilon}\left(x, t_{0}\right) & \text { if } x \in B_{5 M \varepsilon^{2}}(0), \\
\delta_{\varepsilon} \equiv u_{t}\left(0, t_{0}\right)+C_{1} \varepsilon^{2} / d^{p q} \geq u_{\varepsilon, t}\left(x, t_{0}\right) & \text { if } x \in B_{5 M \varepsilon^{2}}(0),
\end{array}
$$


if $C_{0}, C_{1}$ are sufficiently large positive constants. Let $W_{\varepsilon}(t)$ be the solution of

$$
\begin{gathered}
W_{\varepsilon}^{\prime \prime}=f(W) \quad \text { if } t>t_{0}, \\
W_{\varepsilon}\left(t_{0}\right)=\gamma_{\varepsilon}, \quad W_{\varepsilon}^{\prime}\left(t_{0}\right)=\delta_{\varepsilon} .
\end{gathered}
$$

Set $U_{\varepsilon}(x, t)=u_{\varepsilon}(\varepsilon x, t)$. From the integral representation (3.1) and a comparison argument (as in [2]) we see that if

$$
\begin{aligned}
W_{\varepsilon}^{\prime}\left(t_{0}\right)+\left(t-t_{0}\right) W_{\varepsilon}\left(t_{0}\right)> & U_{\varepsilon, t}\left(x, t_{0}\right)+\left(t-t_{0}\right) U_{\varepsilon}\left(x, t_{0}\right) \\
& +\left(t-t_{0}\right)\left|\nabla_{x} U_{\varepsilon}\left(x, t_{0}\right)\right|
\end{aligned}
$$

then

$$
W_{\varepsilon}(t)>u_{\varepsilon}(x, t) \quad \text { in } K
$$

and consequently

$$
\phi_{\varepsilon}(0)>T_{\varepsilon}
$$

where $T_{\varepsilon}$ is the blow-up time of $W_{\varepsilon}(t)$. Since $t-t_{0} \leq 5 M \varepsilon$ and $\left|\nabla_{x} U(x, t)\right|=$ $\varepsilon\left|\nabla u_{\varepsilon}(\varepsilon x, t)\right|,(5.10)$ is a consequence of Lemma 5.2 provided we choose $C_{1}$ to be sufficiently large (independently of $\varepsilon$ ).

Setting $\gamma=u\left(0, t_{0}\right), \delta=u_{t}\left(0, t_{0}\right)$ and using (2.3), we compute that

$$
\begin{aligned}
\phi(0)-T_{\varepsilon}= & \int_{\gamma}^{\infty}\left[2 F(u)-2 F(\gamma)+\delta^{2}\right]^{-1 / 2} d u \\
& -\int_{\gamma_{\varepsilon}}^{\infty}\left[2 F(u)-2 F\left(\gamma_{\varepsilon}\right)+\delta_{\varepsilon}^{2}\right]^{-1 / 2} \\
\leq & \int_{\gamma}^{\gamma_{\varepsilon}}\left[2 F(u)-2 F(\delta)+\delta^{2}\right]^{1 / 2} d u \\
& +\int_{\gamma_{\varepsilon}}^{\infty} \frac{C \varepsilon^{2} d^{-(3 p+1) /(p-1)}}{\left[2 F(u)-2 F(\gamma)+\delta^{2}\right]^{3 / 2}} \equiv I_{1}+I_{2}
\end{aligned}
$$

Clearly

$$
I_{1} \leq C\left(\gamma_{\varepsilon}-\gamma\right) / \delta \leq C \varepsilon^{2} .
$$

Next, substituting $u=\gamma v$ into $I_{2}$, we get

$$
I_{2} \leq \frac{C \gamma}{\gamma^{(p+1) 3 / 2}} \frac{\varepsilon^{2}}{d^{(3 p+1) /(p-1)}} \leq C \varepsilon^{2} .
$$

We have thus proved that $\phi(0)-T_{\varepsilon} \leq C \varepsilon^{2}$. Combining this with (5.10), it follows that

$$
\phi(0)-\phi_{\varepsilon}(0) \leq C \varepsilon^{2} .
$$

Similarly one proves that $\phi(0)-\phi_{\varepsilon}(0) \geq-C \varepsilon^{2}$, and thus $\left|\phi(0)-\phi_{\varepsilon}(0)\right| \leq C \varepsilon^{2}$. Since the above proof applies about each point $x$ in some neighborhood of $x=0$, (5.8) follows.

6. Estimating $\nabla\left(\phi_{\varepsilon}-\phi\right)$. Denote by $\phi_{m}(x)$ and $\psi_{m}(x)$ the solutions of

$$
u\left(x, \phi_{m}(x)\right)=m, \quad u_{t}\left(x, \psi_{m}(x)\right)=m
$$

in view of Lemma 2.2, $\phi_{m}(x)$ and $\psi_{m}(x)$ are well defined for $x \in B_{R}$, provided $m$ is sufficiently large; $R$ is as usual a small enough positive number. Denote by 
$N(x), N_{m}(x)$ and $M_{m}(x)$ the unit normals in the positive $t$-direction of the surfaces $\{t=\phi(x)\},\left\{t=\phi_{m}(x)\right\}$ and $\left\{t=\psi_{m}(x)\right\}$ respectively. Thus, for instance,

$$
N_{m}(x)=\left[1+\left|\nabla \phi_{m}(x)\right|^{2}\right]^{-1 / 2}\left(-\nabla \phi_{m}(x), 1\right) \text {. }
$$

For any $\eta>0$, denote by $S_{\eta}(x)$ the set of all unit vectors $\tau=\tau(x)$ with

$$
\tau \cdot N(x) \geq \eta \text {. }
$$

We claim

$$
\left|\nabla\left(\phi_{m}(x)-\phi(x)\right)\right| \leq C m^{-(3 p+1) / 2} \quad \text { if } x \in B_{R} .
$$

Indeed, analogously to (2.9) $\left(t_{0}\right.$ is taken close to $\left.\phi(0)\right) \phi_{m}(x)$ is given by

$$
\phi_{m}(x)=t_{0}+\int_{u\left(x, t_{0}\right)}^{m} \frac{d u}{\left[2 F(u)-2 F\left(u\left(x, t_{0}\right)\right)+\left(u_{t}\left(x, t_{0}\right)\right)^{2}\right]^{1 / 2}} .
$$

Therefore

$$
\begin{aligned}
\nabla \phi_{m}(x)= & -\frac{\nabla u\left(x, t_{0}\right)}{u_{t}\left(x, t_{0}\right)} \\
& +\int_{u\left(x, t_{0}\right)}^{m} \frac{\left[f\left(u\left(x, t_{0}\right)\right) \nabla u\left(x, t_{0}\right)-u_{t}\left(x, t_{0}\right) \nabla u_{t}\left(x, t_{0}\right)\right] d u}{\left[2 F(u)-2 F\left(u\left(x, t_{0}\right)\right)+\left(u_{t}\left(x, t_{0}\right)\right)^{2}\right]^{3 / 2}} .
\end{aligned}
$$

Since $\phi(x)=\phi_{\infty}(x)$, we deduce that

$$
\nabla\left(\phi(x)-\phi_{m}(x)\right)=\int_{m}^{\infty}[\cdots] d u
$$

with the same integrand as in the preceding integral. Hence

and (6.3) follows.

$$
\left|\nabla\left(\phi_{m}(x)-\phi(x)\right)\right| \leq C \int_{m}^{\infty} \frac{d u}{u^{(p+1) 3 / 2}}
$$

We shall next prove that

$$
\left|\nabla\left(\psi_{m}(x)-\phi(x)\right)\right| \leq C m^{-(3 p+1) /(p+1)} \quad \text { if } x \in B_{R} .
$$

Indeed, we have

$$
u_{t}^{2}-u_{t}^{2}\left(x, t_{0}\right)+2 F\left(u\left(x, t_{0}\right)\right)=2 F(u)
$$

and $F(u)$ has an inverse $G=F^{-1}$, well defined and smooth, for all $u=u(x, t)$ with $t>t_{0}$ (by $(2.6),(2.7)$ ). We can then write

$$
u=G\left(\frac{1}{2} u_{t}^{2}-\frac{1}{2} u_{t}^{2}\left(x, t_{0}\right)+F\left(u\left(x, t_{0}\right)\right)\right),
$$

and (1.7) takes the form

By integration we then obtain

$$
u_{t t}=f\left(G\left(\frac{1}{2} u_{t}^{2}-\frac{1}{2} u_{t}^{2}\left(x, t_{0}\right)+F\left(u\left(x, t_{0}\right)\right)\right)\right) .
$$

$$
\psi_{m}(x)=t_{0}+\int_{u_{t}\left(x, t_{0}\right)}^{m} \frac{d v}{f\left(G\left(\frac{1}{2} v^{2}+a(x)\right)\right)}
$$

where

$$
a(x)=-\frac{1}{2} u_{t}^{2}\left(x, t_{0}\right)+F\left(u\left(x, t_{0}\right)\right) .
$$

The same formula holds for $\phi(x)$ with $m=\infty$. Taking the gradient of the difference, we get

$$
\left|\nabla\left(\psi(x)-\psi_{m}(x)\right)\right| \leq C \int_{m}^{\infty} \frac{d v}{\left(\frac{1}{2} v^{2}+a(x)\right)^{1+p /(p+1)}}
$$

and (6.4) follows. 
LEMMA 6.1. There exist a positive constant $c$ such that, for any $\eta \in(0,1)$, if

$$
\phi(x)-t<c \eta^{(p-1) /(3 p+1)}
$$

then

$$
\begin{gathered}
\tau \cdot N_{u(x, t)}(x)>\eta / 3 \\
\tau \cdot M_{u_{t}(x, t)}(x)>\eta / 3
\end{gathered}
$$

for any $x \in B_{R}, \tau \in S_{2 \eta / 3}(x)$.

ProOF. From (6.3), (6.4) and Lemma 2.2,

$$
\begin{aligned}
& \text { (6.8) } \quad\left|\nabla\left(\phi_{m}-\phi\right)\right| \leq C / u^{(3 p+1) / 2} \leq C(\phi-t)^{(3 p+1) /(p-1)}, \quad m=u(x, t), \\
& (6.9) \quad\left|\nabla\left(\psi_{m}-\phi\right)\right| \leq C / u_{t}^{(3 p+1) /(p+1)} \leq C(\phi-t)^{(3 p+1) /(p-1)}, \quad m=u_{t}(x, t) .
\end{aligned}
$$

From (6.1) we have

$$
\left|N(x)-N_{u(x, t)}(x)\right| \leq C\left|\nabla\left(\phi-\psi_{m}\right)(x)\right| .
$$

Using (6.8) and (6.5) we get

$$
\left|N(x)-N_{u(x, t)}(x)\right| \leq C(\phi(x)-t)^{(3 p+1) /(p-1)} \leq C c \eta<\eta / 3
$$

if $c<1 /(3 C)$; thus (6.6) follows. The proof of (6.7) follows similarly, making use of $(6.9)$.

LEMMA 6.2. There exist positive constants $c_{0}, c_{1}, C_{0}$ such that for any $\eta \in$ $(0,1)$ such that

$$
\varepsilon^{2}<c_{0} \eta
$$

the following is true: if

$$
\begin{aligned}
2 M \varepsilon & \leq \phi(x)-t<c \eta^{(p-1) /(3 p+1)}, \\
\tau & \in S_{2 \eta / 3}(x), \quad x \in B_{R},
\end{aligned}
$$

then

$$
\begin{aligned}
& \frac{\partial u_{\varepsilon}(x, t)}{\partial \tau} \geq \frac{c_{1} \eta}{(\phi(x)-t)^{p q-1}} \\
& \frac{\partial}{\partial t} \frac{\partial u_{\varepsilon}(x, t)}{\partial \tau} \geq \frac{c_{1} \eta}{(\phi(x)-t)^{p q}} \\
& \left|\nabla \frac{\partial u_{\varepsilon}(x, t)}{\partial \tau}\right| \leq \frac{C_{0}}{(\phi(x)-t)^{p q}}
\end{aligned}
$$

provided $\varepsilon$ is sufficiently small.

ProOF. The estimate (6.15) follows from Lemma 5.2. Next, from Lemma 6.1 we have, if $\tau \in S_{2 \eta / 3}(x)$,

$$
\frac{\partial u(x, t)}{\partial \tau} \geq \frac{\eta}{3} \frac{\partial u}{\partial N_{u}}, \quad \frac{\partial u}{\partial t} \frac{\partial u(x, t)}{\partial \tau} \geq \frac{\eta}{3} \frac{\partial u_{t}}{\partial M_{u_{t}}} .
$$


We also clearly have

$$
\frac{\partial u}{\partial t}=\alpha \frac{\partial u}{\partial N_{u}}, \quad \frac{\partial^{2} u}{\partial t^{2}}=\beta \frac{\partial u_{t}}{\partial M_{u_{t}}}
$$

where

$$
\begin{array}{ll}
\alpha=\alpha(x, t)=\left[1+\left|\nabla \phi_{m}(x)\right|^{2}\right]^{-1 / 2}, & m=u(x, t), \\
\beta=\beta(x, t)=\left[1+\left|\nabla \psi_{m}(x)\right|^{2}\right]^{-1 / 2}, & m=u_{t}(x, t) .
\end{array}
$$

Recalling the estimates in (2.11), we conclude that

$$
\frac{\partial u}{\partial \tau} \geq \frac{c \eta}{(\phi-t)^{p q-1}}, \quad \frac{\partial}{\partial t} \frac{\partial u}{\partial \tau} \geq \frac{c \eta}{(\phi-t)^{p q}} \quad(c>0) .
$$

If we now make use of Lemma 5.3, we obtain from the last two inequalities the inequalities (6.13), (6.14) provided $\varepsilon^{2} / \eta$ is bounded by a sufficiently small constant, i.e., provided (6.10) holds with $c_{0}$ small enough (independently of $\eta, \varepsilon$ ). This completes the proof of the lemma.

We now proceed to establish (1.17). Fix a point $y$ in $B_{R}(R$ small $)$ and let $t_{1}=\phi(y)-3 M \varepsilon$. Denote by $K$ the cone with base

$$
B \equiv\left\{\left(x, t_{1}\right) ;|x-y|<5 M \varepsilon^{2}\right\}
$$

and vertex $(y, \phi(y)+2 M \varepsilon)$.

If $\tau \in S_{\eta}(y)$ then (since $\phi$ is smooth) $\tau \in S_{2 \eta / 3}(x)$ for any $x \in B_{5 M \varepsilon^{2}}(y)$ provided

$$
\varepsilon^{2} \leq \eta / C
$$

and $C$ is a sufficiently large positive constant. It follows that (6.13)-(6.15) hold on $B$. We can therefore apply a comparison argument to $U_{\varepsilon}(x, t)=\partial u_{\varepsilon}(y+\varepsilon x, t) / \partial \tau$ (as in [2]; cf. also the proof of Lemma 5.4) and deduce that

$$
\partial u_{\varepsilon} / \partial \tau>0 \text { in } K \cap\{t<\phi\}
$$

provided (6.16) holds with $C$ large enough. Since $K$ contains $\left(y, \phi_{\varepsilon}(y)\right)$ in its interior, we see from (6.17) that $u_{\varepsilon}$ is increasing along any direction $\tau\left(\tau \in S_{\eta}(y)\right)$, in some neighborhood of $\left(y, \phi_{\varepsilon}(y)\right)$. This means that the direction of $\nabla \phi_{\varepsilon}(y)$ and $\nabla \phi(y)$ differ by at most $\eta$. Thus

$$
\left|\nabla\left(\phi_{\varepsilon}-\phi\right)\right| \leq \eta \quad \text { at } y .
$$

The constant $\eta$ was subject only to the constraints $(6.10),(6.11)$ for any $x \in$ $B_{5 M \varepsilon^{2}}(y)$ with $\phi(y)-t=3 M \varepsilon$, and (6.16). Thus we can choose $\eta=C \varepsilon^{2}$, where $C$ is a sufficiently large positive constant, and then (1.17) follows.

REMARK 6.1. Using Lemma 5.4 we can extend the estimates of Lemma 5.3 to $t_{1}<t<\phi(x)-M \varepsilon^{2}$, and of Lemma 6.2 under the condition $2 M \varepsilon^{2}<\phi(x)-t<$ $c \eta^{(p-1) /(3 p+1)}$ instead of (6.11). If we now follow the proofs of $(1.16),(1.17)$ with these improved lemmas (using, for instance, in the proof of (1.17), cones with base $B$ on $t=t_{1} \equiv \phi(y)-3 M \varepsilon^{2}$ given by $\left.B=\left\{\left(x, t_{1}\right) ;|x-y|<5 M \varepsilon^{3}\right\}\right)$, we do not get any improvements of the estimates (1.16), (1.17).

7. The case $\phi(0)=\infty$. In this section we consider the case where $\phi(0)=\infty$ but $\phi(x)<\infty$ if $x \in B_{R} \backslash\{0\}$ for some $R>0$. By Lemma 1.3 for any $x_{0} \in B_{R} \backslash\{0\}$ there exists $\delta_{0}=\delta_{0}\left(\left|x_{0}\right|\right)$ and $\varepsilon_{0}=\varepsilon_{0}\left(\delta_{0},\left|x_{0}\right|\right)$ positive such that there is a unique solution $u_{\varepsilon}(x, t)$ with blow-up surface $t=\phi_{\varepsilon}(x)$ if $x \in B_{\delta}(x, 0)$ and $0<\varepsilon<\varepsilon_{0}$; 
however $\varepsilon_{0}$ may go to zero if $x_{0} \rightarrow 0$. On the other hand, for $N=1, \phi_{\varepsilon}$ is finite for all $x \in B_{R}$ or even for all $x \in \mathbf{R}^{1}$ with $\left|\phi_{\varepsilon}^{\prime}(x)\right|<1 / \varepsilon$ (by [1]).

For simplicity we take

$$
f(u)=\left(u^{+}\right)^{p}, \quad p>1,
$$

and consider first the case where

$$
\begin{aligned}
& g(x)>0 \quad \text { in } B_{R} \backslash\{0\}, \quad g(x)=\sum_{i=1}^{N} \alpha_{i} x_{i}^{2}+O\left(|x|^{3}\right) \quad \text { where } \alpha_{i}>0 \forall i, \\
& h(x)>0 \quad \text { in } B_{R} \backslash\{0\}, \quad h(x)=\sum_{i=1}^{N} \beta_{i} x_{i}^{2}+O\left(|x|^{3}\right) \quad \text { where } \beta_{i}>0 \forall i .
\end{aligned}
$$

We further assume that if $N=2$ or $N=3$ then the solution $u_{\varepsilon}(x, t)$ with finitevalued blow-up surface $t=\phi_{\varepsilon}(x)$ exist for all $x \in B_{R}$.

THEOREM 7.1. Under the foregoing assumptions, there exist positive constants $c_{0}, c_{1}$ such that

$$
c_{0} \varepsilon^{-\sigma} \leq \phi_{\varepsilon}(x) \leq c_{1} \varepsilon^{-\sigma} \quad \text { where } \sigma=2(p-1) / 3 p-1 .
$$

PROOF. We shall construct a subsolution $v_{\varepsilon}(x, t)$ with blow-up surface $t=\psi_{\varepsilon}(x)$ such that

$$
\psi_{\varepsilon}(0)=c_{1} \varepsilon^{-\sigma}
$$

Similarly we shall construct a supersolution $w_{\varepsilon}(t)$ with blow-up time $T_{\varepsilon}$,

$$
T_{\varepsilon} \geq c_{0} \varepsilon^{-\sigma} \text {. }
$$

Since $w_{\varepsilon} \geq u_{\varepsilon}>v_{\varepsilon}$

$$
\psi_{\varepsilon}(0) \geq T_{\varepsilon} \geq c_{0} \varepsilon^{-\sigma},
$$

and the proof of (6.4) will be completed.

Observe that the domain of dependence of a point $(0, T)$ with $T \sim c \varepsilon^{-\sigma}$ is a cone whose base on $\{t=0\}$ is $B_{R_{0}}$ where $R_{0} \sim c \varepsilon^{1-\sigma}$. Therefore, in constructing $v_{\varepsilon}$ and $w_{\varepsilon}$ we need to define their Cauchy data only in a ball $\left\{|x|<C \varepsilon^{1-\sigma}\right\}$ with an appropriate positive constant $C$.

We take

$$
v_{\varepsilon}(x, t)=(1+t) \delta|x|^{2}+k(t)
$$

where $\delta$ is a small positive constant and $k$ is the solution of

$$
k^{\prime \prime}=k^{p}+2 \delta N \varepsilon^{2} t \quad \text { if } t>0, \quad k(0)=k^{\prime}(0)=0 .
$$

It is easy to check that $\square_{\varepsilon} v_{\varepsilon} \leq v_{\varepsilon}^{p}$. In order to compare the Cauchy data, we work with $U_{\varepsilon}(x, t)=u_{\varepsilon}(\varepsilon x, t)$ and $V_{\varepsilon}(x, t)=v_{\varepsilon}(\varepsilon x, t)$. Denote by $G_{\varepsilon}$ the function defined by (3.3) with $g=g(\varepsilon x), h=h(\varepsilon x)$, and denote by $\tilde{G}$ the function defined by (3.3) with $g=h=\delta \varepsilon^{2}|x|^{2}$. Using the integral representations (3.1) for $U_{\varepsilon}$ and $V_{\varepsilon}$, and noting that $G \geq \tilde{G}$ if $\delta$ is small enough, we deduce by comparison (cf. [2]) that $U_{\varepsilon} \geq V_{\varepsilon}$, i.e., $u_{\varepsilon} \geq v_{\varepsilon}$. 
The function $K(t)=\lambda^{2 /(1-p)} k(\lambda t)$ where $\lambda=\varepsilon^{-\sigma}$ satisfies

$$
K^{\prime \prime}=K^{p}+2 \delta N t, \quad K(0)=K^{\prime}(0)=0
$$

and it blows up in some finite time $c_{1}$. Therefore $k(t)$ blows up in time $c_{1} \varepsilon^{-\sigma}$, and (7.5) follows.

We next construct a supersolution $w_{\varepsilon}(t)$,

$$
\begin{aligned}
w_{\varepsilon}^{\prime \prime} & =w_{2}^{p} \quad \text { for } t>0, \\
w_{\varepsilon}(0) & =w_{\varepsilon}^{\prime}(0)=A \varepsilon^{2(1-\sigma)}, \quad A>0 .
\end{aligned}
$$

If $A$ is sufficiently large then, using the representation (3.1) for $U_{\varepsilon}(x, t)$ and $w_{\varepsilon}(t)$ we deduce that $w_{\varepsilon} \geq u_{\varepsilon}$ in $K^{\varepsilon}(0, T)$ for $T=c_{1} \varepsilon^{-\sigma}$. Thus it remains to prove that $w_{\varepsilon}$ blows up in time $T_{\varepsilon} \geq c_{0} \varepsilon^{-\sigma}$ for some $0<c_{0}<c_{1}$.

Now, from (7.10) we get, by integration,

$$
\frac{1}{2} w_{\varepsilon, t}^{2}=\frac{w_{\varepsilon}^{p+1}}{p+1}+\frac{A^{2}}{2} \varepsilon^{4(1-\sigma)}(1+o(1))
$$

where $o(1) \rightarrow 0$ if $\varepsilon \rightarrow 0$; hence

$$
T_{\varepsilon}=\int_{A \varepsilon^{2(1-\sigma)}}^{\infty} \frac{d w}{\left[2 w^{p+1} /(p+1)+A^{2} \varepsilon^{4(1-\sigma)}\right]^{1 / 2}} \geq c_{0} \varepsilon^{-\sigma} .
$$

We shall next consider the case where $g$ and $h$ vanish to higher order at $x=0$. We take $N=1$ and assume that

$$
g(x) \geq 0, g^{\prime \prime}(x) \geq 0 \text { for }|x| \text { near } 0
$$

and

$$
\begin{aligned}
h(x) & =\beta|x|^{n}+O\left(|x|^{n+1}\right), \\
h^{\prime}(x) & =n \beta|x|^{n-2} x+O\left(|x|^{n}\right), \quad \beta>0,
\end{aligned}
$$

where $n$ is a positive number $\geq 2$.

Set

$$
\sigma=\{1+(p+1) / n(p-1)\}^{-1} .
$$

THEOREM 7.2. Let $N=1$ and let (7.1), (7.11), (7.12) hold. Then there exist positive constants $c_{0}, c_{1}$ such that (7.4) holds with $\sigma$ given by (7.13).

Notice that for $n=2$ this is an improvement of Theorem 7.1 for $N=1$, since the condition (7.11) is weaker than (7.2).

PROOF. We represent $u_{\varepsilon}$ in the form

$$
\begin{aligned}
u_{\varepsilon}(x, t)= & \frac{1}{2}[g(x+\varepsilon t)+g(x-\varepsilon t)]+\frac{1}{2 \varepsilon} \int_{x-\varepsilon t}^{x+\varepsilon t} h(y) d y \\
& +\frac{1}{2 \varepsilon} \int_{0}^{t} \int_{x-\varepsilon(t-\tau)}^{x+\varepsilon(t-\tau)}\left(u^{+}\right)^{p}(y, \tau) d y d \tau .
\end{aligned}
$$

By differentiation,

$$
\begin{aligned}
u_{\varepsilon, t}(x, t)= & \frac{\varepsilon}{2}\left[g^{\prime}(x+\varepsilon t)-g^{\prime}(x-\varepsilon t)\right]+\frac{1}{2}[h(x+\varepsilon t)+h(x-\varepsilon t)] \\
& +\frac{1}{2} \int_{0}^{t}\left[\left(u^{+}\right)^{p}(x+\varepsilon(t-\tau), \tau)+\left(u^{+}\right)^{p}(x-\varepsilon(t-\tau), \tau)\right] d \tau .
\end{aligned}
$$


Using (7.14) and the assumption $g(x) \geq 0$ for $x$ near 0 , we get

$$
u_{\varepsilon}\left(x, \varepsilon^{-\sigma}\right) \geq \frac{1}{2 \varepsilon} \int_{x-\varepsilon^{1-\sigma}}^{x+\varepsilon^{1-\sigma}} h(y) d y \geq \beta \varepsilon^{-\sigma} \varepsilon^{n(1-\sigma)} \quad \text { if }|x|<R
$$

provided $R$ is small; $\alpha$ is some positive constant.

Next, since $g^{\prime \prime} \geq 0$, we obtain from (7.15),

$$
u_{\varepsilon, t}\left(x, \varepsilon^{-\sigma}\right) \geq \beta \varepsilon^{n(1-\sigma)} / 4 \text { if }|x| \leq R .
$$

We now compare $u_{\varepsilon}\left(x, \varepsilon^{-\sigma}+t\right)$ with the solution $v_{\varepsilon}(t)$ of

$$
\begin{aligned}
v_{\varepsilon}^{\prime \prime} & =v_{\varepsilon}^{p}, \quad t>0, \\
v_{\varepsilon}(0)=v_{\varepsilon}^{\prime}(0) & =\gamma_{0} \varepsilon^{n(1-\sigma)}, \quad \gamma_{0}=\beta / 4 .
\end{aligned}
$$

This solution blows up at time $T\left(\gamma_{0} \varepsilon^{n(1-\sigma)}, \gamma_{0} \varepsilon^{n(1-\sigma)}\right)$ where $T(\gamma, \delta)$ is defined by (2.3). It is easily computed that the blow-up time is bounded from above by

$$
C\left(\varepsilon^{-n(1-\sigma)}\right)^{(p-1) /(p+1)} \quad(C \text { positive constant })
$$

which is equal to $C \varepsilon^{-\sigma}$ (with another positive constant $C$ ), by the definition of $\sigma$ in (7.13). Hence

$$
\phi_{\varepsilon}(0)<\varepsilon^{-\sigma}+C_{*} \varepsilon^{-\sigma}=c_{1} \varepsilon^{-\sigma} \quad\left(c_{1}=1+C\right) .
$$

In order to estimate $\phi_{\varepsilon}(0)$ from below it is sufficient (in view of (7.18)) to use the initial data only in the interval $\left\{|x|<c_{1} \varepsilon^{1-\sigma}\right\}$. Thus we can use the same function $w_{\varepsilon}$ as in (7.10) for a supersolution. Proceeding as in the argument following (7.10), we then derive the lower estimate in (7.4) with $\sigma$ as in (7.13).

REMARK. The above proof extends to $N=2,3$ and say

$$
g(x) \sim \alpha|x|^{n}, \quad h(x) \sim \beta|x|^{n} \quad(\alpha>0, \beta>0)
$$

provided we already know that

$$
u_{\varepsilon}(x, t) \geq 0, \quad u_{\varepsilon, t}(x, t) \geq 0
$$

for $|x| \leq C \varepsilon^{1-\sigma}, 0 \leq t \leq \varepsilon^{-\sigma}$; we use here the representation (3.1) for both $u_{\varepsilon}$ and $u_{\varepsilon, t}$. However the usual method for proving (7.20) (by using the representation (3.1), as in [2]) does not extend to the present situation where (7.19) holds, even for $t$ small.

\section{REFERENCES}

1. L. A. Caffarelli and A. Friedman, Differentiality of the blow-up curve for one dimensional nonlinear wave equations, Arch. Rational Mech. Anal. 91 (1985), 83-98.

2. __ The blow-up boundary for nonlinear wave equations, Trans. Amer. Math. Soc. 297 (1986), 223-241.

3. R. Glassey, Blow-up theorems for nonlinear wave equations, Math. Z. 132 (1973), 183-203.

4. __ Finite-time blow-up for solutions of nonlinear wave equations, Math. Z. 177 (1981), 323340.

5. F. John, Blow-up of solutions of nonlinear wave equations in three space dimensions, Manuscripta Math. 28 (1979), 235-268. 
6. H. Levine, Instability and nonexistence of global solutions to nonlinear wave equations of the form $P u_{t t}=-A u+F(u)$, Trans. Amer. Math. Soc. 192 (1974), 1-21.

7. T. Kato, Blow-up of solutions of some nonlinear hyperbolic equations, Comm. Pure Appl. Math. 32 (1980), 501-505.

Department of MAthematics, University of Minnesota, Minneapolis, MinNESOTA 55455

Department of Mathematics, Purdue University, West Lafayette, Indiana 47907 